\title{
Spatially resolved investigations of lifetime enhancement in vertically grown, multicrystalline silicon ribbons
}

\author{
P. Geiger*, G. Kragler, G. Hahn, P. Fath \\ Universität Konstanz, Fachbereich Physik, Fach X916, 78457 Konstanz, Germany
}

\begin{abstract}
The influence of gettering or defect passivation steps on recombination activity in the vertically grown, multicrystalline ribbon materials edge-defined film-fed growth and string ribbon silicon has been investigated with the help of photoconductance decay. In contrast to well-known results of integral measurements, spatially resolved lifetime mappings have been obtained by applying microwave detection technique.

This aspect of spatial resolution has been found to be indispensable for investigating the impact of different processing steps on material quality in an accurate way. Apart from strong variations in as-grown lifetimes that have been found throughout vertically grown silicon wafers, this is due to areas of comparable starting lifetimes which have been revealed to react very differently to applied processing steps. After processing, some of them reach minority charge carrier lifetimes of more than $300 \mu$ s whereas others just show values of a few microseconds. As a consequence, the results of integral measurements strongly depend on the nature of areas incorporated in the specific sample. An impression of the corresponding uncertainties inherent to integral measurements has been obtained by statistical evaluation of spatially resolved lifetime data.
\end{abstract}

Keywords: Ribbon silicon; Lifetime measurement; Gettering; Hydrogen passivation

Corresponding author. Now with Patentanwalte/patent attorneys Westphal, Mussgnug \& Partner, Am Riettor 5, 78048 Villingen-Schwenningen, Germany. Tel.: +49-7531-88-3644; fax: + 49-7531-88-3895.

E-mail address: geiger@wemupat.de (P. Geiger). 


\section{Introduction}

The production of conventionally cast multicrystalline (mc) silicon wafers requires expensive wafering steps causing $24 \%$ of the final fabrication costs per Watt-peak [1]. Silicon ribbons, instead, are grown directly out of the melt in the required thickness, so that cost-intensive wafering steps and related material losses can be avoided. Compared to cast silicon, the resulting cost-advantages should be above $20 \%$, referring to the fabrication costs per Watt-peak [1].

The most promising materials in the field of silicon ribbons are edge-defined filmfed growth (EFG) silicon, commercially produced by RWE Solar Inc. (formerly ASE Americas Inc.), string ribbon (SR) silicon industrially fabricated by Evergreen Solar Inc., dendritic web silicon of Ebara Solar Inc., ribbon growth on substrate (RGS) silicon fabricated by ECN and silicon-film sheets by AstroPower Inc. RGS silicon as well as silicon-film sheets are grown horizontally, i.e. the pulling direction is perpendicular to the crystallization direction. Furthermore, the vertically grown Dentritic Web silicon shows a moncrystalline structure. Therefore, these two ribbon materials have not been investigated in this study, where we focused on the multicrystalline and vertically grown EFG and SR ribbons. The specific growth procedures of these two materials result in grain structures and structural defect distributions, which are significantly different from those in standard multicrystalline cast silicon. The fabrication of solar cells consequently requires the development of processing schemes specifically adapted to these materials. A possible way of studying the impact of various processing steps on ribbon materials is to measure minority charge carrier lifetimes before and after the application of a specific processing step. Intensive research in this field has already been done with the help of integral lifetime measurements [2-4]. Former investigations, however, have shown quite strong variations of material quality within a few square centimetres, so that it is not obvious that regions of different quality react in the same way and to the same extent to a certain processing step [5,6]. For this reason, we have investigated the impact of various processing steps on EFG and SR silicon in a spatially resolved way.

\section{Experimental approach}

\subsection{Bulk lifetime mapping and visualization}

The minority charge carrier lifetimes of the ribbon silicon samples, each covering an area of $5 \times 5 \mathrm{~cm}^{2}$, were mapped using the method of microwave detected photoconductance decay ( $\mu$-PCD). All measurements were performed under low injection conditions and bias light (one sun) using a laser wavelength of $905 \mathrm{~nm}$ for charge carrier generation. In this way an effective lifetime value $\tau_{\text {eff }}$ has been measured which represents the sum of the inverse bulk lifetime $\tau_{\mathrm{b}}$ and an inverse surface lifetime $\tau_{\mathrm{s}}$ as illustrated in Eq. (1).

$$
\frac{1}{\tau_{\mathrm{eff}}}=\frac{1}{\tau_{\mathrm{b}}}+\frac{1}{\tau_{\mathrm{s}}}=\frac{1}{\tau_{\mathrm{b}}}+\alpha^{2} D_{\mathrm{n}}
$$


The surface related part can be calculated with the help of the electron diffusion constant $D_{\mathrm{n}}$ and $\alpha$, which is the solution of the transcendental equation given in Eq. (2) that contains the wafer thickness $d$ and the effective surface recombination velocity $S_{\text {eff }}$.

$$
\tan \left(\frac{\alpha d}{2}\right)=\frac{S_{\mathrm{eff}}}{\alpha D_{\mathrm{n}}} .
$$

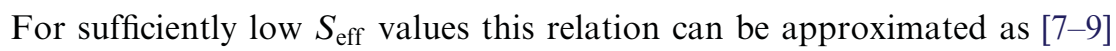

$$
\alpha^{2}=\frac{2 S_{\mathrm{eff}}}{d D_{\mathrm{n}}} .
$$

As we were interested in the bulk lifetime $\tau_{\mathrm{b}}$, it was therefore necessary to reduce $S_{\text {eff }}$, so that the contribution of the surface lifetime to $\tau_{\text {eff }}$ could be neglected. This was achieved by using an iodine-ethanol solution for reproducible passivation of the wafer surfaces [10]. In this way it was possible to investigate the impact and synergetic effects of different processing steps on the same wafer by subsequently measuring the resulting bulk lifetime alterations.

The determination of $\tau_{\text {eff }}$ or $\tau_{\mathrm{b}}$ from the photoconductance decay requires the selection of a time range in which the decay of the photoconductance after the end of the laser pulse is evaluated. The adequate time range for a specific wafer depends on the material properties. Due to the strong variations of material quality within EFG or SR silicon, however, it is not possible to choose a single time range that is well suited for the whole sample. This problem was addressed by measuring the wafer several times partly or as a whole with different time ranges. In the following, the resulting data has been combined by extracting from each measurement just those data points for which the time range was well suited, and putting them in a new mapping together with the reliable data points of the other measurements. As a consequence, rather high lifetimes as well as quite poor ones are reliable in the finally obtained mapping. All the mappings shown in this study are such combined ones. In this way they differ from the usually shown standard mappings which are normally based on only one single time range.

\subsection{Design of experiment}

For each material the experiment was divided into four processing sequences illustrated by the four columns shown in Fig. 1. At the beginning $20 \mu \mathrm{m}$ were removed on each side of the wafers by an acidic etching step in order to obtain comparable surfaces. Before each $\mu$-PCD measurement indicated in Fig. 1 the samples were chemically cleaned and their surfaces were passivated with an iodine-ethanol solution. The $\mathrm{POCl}_{3}$ emitter diffusion (sheet resistivity $80 \Omega / \mathrm{sq}$ ) was performed in a quartz tube furnace and the aluminium required for a $30 \mathrm{~min}$ gettering step at $800^{\circ} \mathrm{C}$ was evaporated onto one side of the wafers. Before the subsequent lifetime measurements, P-emitter as well as aluminium back surface field (BSF) were etched back using again an acidic etching solution. In this way charge carrier recombination in the emitter or problems with surface passivation on the 


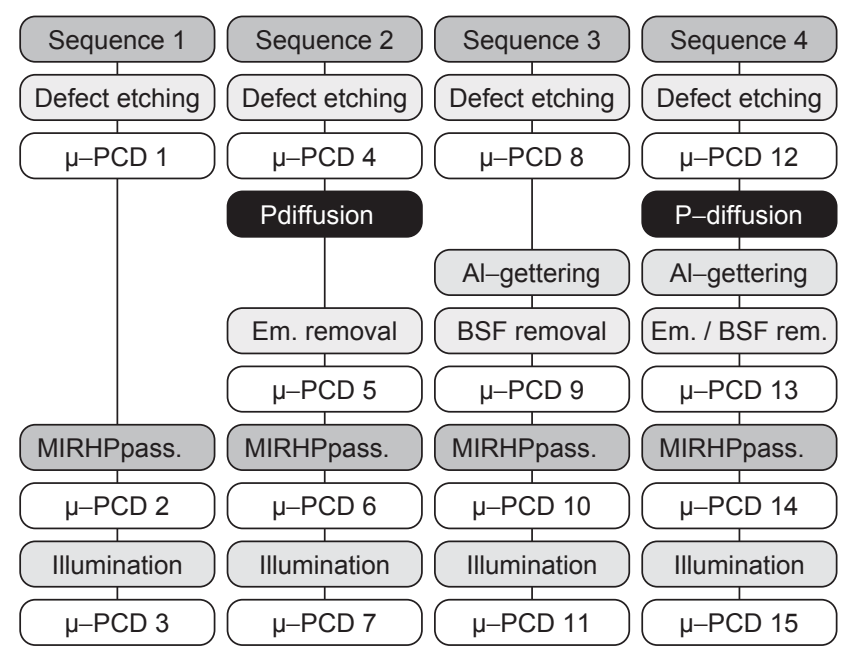

Fig. 1. Structure of the experiment. Spatially resolved bulk lifetime measurements have been performed after various successive processing steps.

back side during the $\mu$-PCD measurements could be avoided. Furthermore, a hydrogen passivation step was incorporated into the different processing sequences, which was realized with the help of a microwave-induced remote hydrogen plasma (MIRHP) [11]. And finally, illumination of the samples (one sun for ten hours) made it possible to examine the stability of this $\mathrm{H}$-passivation.

\section{Results}

\subsection{Obtained mappings}

Each time a $\mu$-PCD measurement is indicated in Fig. 1, several measurements were performed and adequately combined as described in Section 2.1. As a consequence all the finally obtained mappings depicted in the following show reliable high and low lifetime values. The mappings of an EFG and a SR wafer that underwent sequence 2 are shown representatively on the left and on the right side of Fig. 2. Nevertheless, there is a region in mapping (a1) of this figure where the measurement was not totally reliable. Consequently, these points were not taken into account and are marked with grey points. All the other mappings, however, do not show this problem.

For each silicon ribbon the corresponding left column depicts the minority charge carrier bulk lifetimes after the various processing steps of sequence 2 given in Fig. 1. First of all they illustrate strong variations in material quality throughout the EFG as well as the string ribbon wafer. In the case of string ribbon material the lifetime values appear to be significantly higher. But it cannot be concluded that this is true in 
EFG

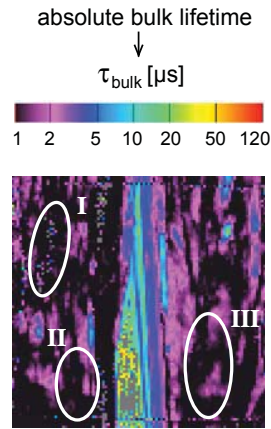

(a1) after defect etching

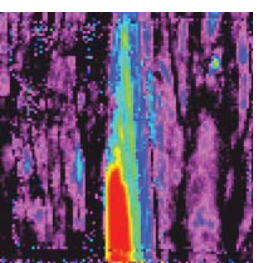

(b1) after P-diffusion

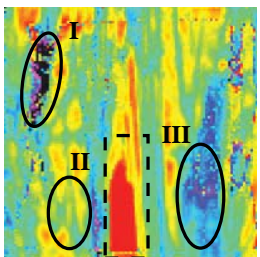

(c1) after MIRHP-pass.

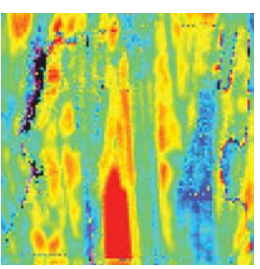

(d1) after illumination
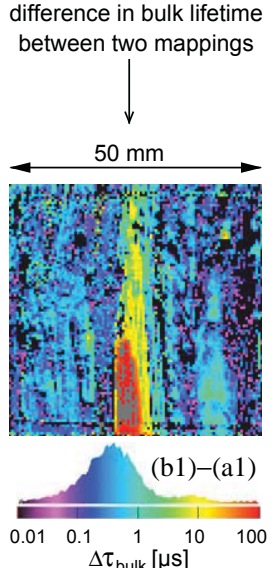

$\tau_{\text {bulk }}[\mu \mathrm{s}]$

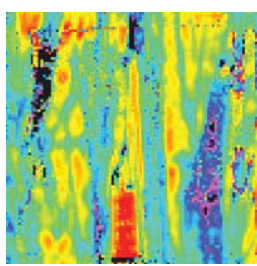

(c1)-(b1)
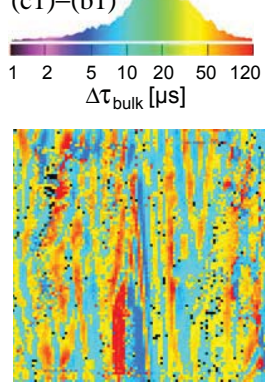

(d1)-(c1)

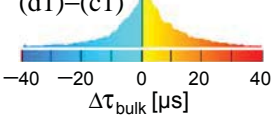

\section{String Ribbon}

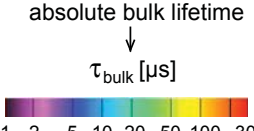

erence in bulk lifetime

between two mappings

$\begin{array}{llllllll}1 & 2 & 5 & 10 & 20 & 50 & 100 & 300\end{array}$

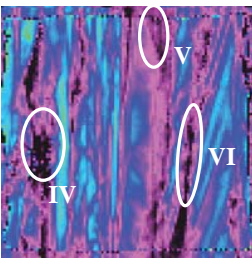

(a2) after defect etching

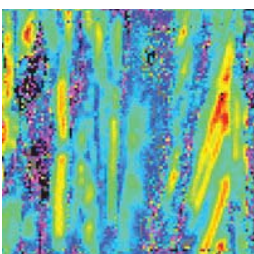

(b2) after $\mathrm{P}$-diffusion

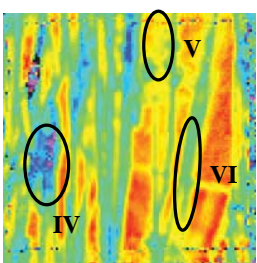

(c2) after MIRHP-pass.

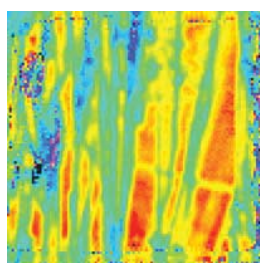

(d2) after illumination
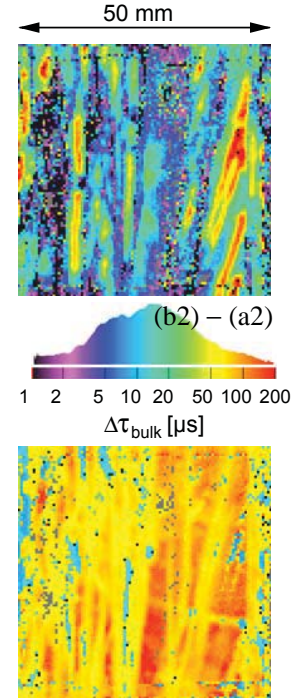

(c2) - (b2)
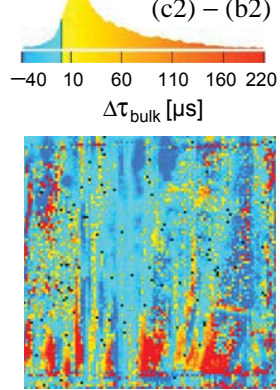

(d2) - (c2)

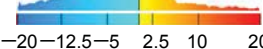

$\Delta \tau_{\text {bulk }}[\mu \mathrm{s}]$

Fig. 2. Bulk lifetime mappings of an EFG (left) and a string ribbon wafer (right), both corresponding to sequence 2 of Fig. 1. Each mapping shows the result of appropriate combinations of various measurements. For each silicon material the left column depicts the bulk lifetimes measured after different processing steps. Differences in lifetime values between two steps are illustrated by the related right columns.

general. The lifetimes measured in such $5 \times 5 \mathrm{~cm}^{2}$ samples depend strongly on the quality of areas incorporated in the specific sample and in both materials areas of rather poor as well as quite good performance can be found. The shares of these 
regions make the difference between these two specific samples. Therefore it is not possible to compare lifetimes in EFG to those in string ribbon silicon on the base of such few samples.

The right column of each material in Fig. 2 illustrates the lifetime alterations caused by the single processing steps as they have been obtained by calculating the difference between the two measurements to the left of each mapping. By this kind of visualization additional information can be gained. Analysing P-gettering, i.e. pictures (a1), (b1) and (b1)-(a1) for EFG or (a2), (b2) and (b2)-(a2) for the case of string ribbon, one finds that in both materials P-gettering leads to strong improvements of partly above $100 \mu$ s in areas of rather high starting lifetimes of about seven or more microseconds. In regions with lower starting lifetimes, instead, enhancements of only some microseconds or even less have been measured. In contrast to P-gettering, MIRHP passivation also effects most areas with worse performance and improves their lifetime values by about 10-20 $\mathrm{s}$. Stronger improvements of $100 \mu \mathrm{s}$ or more are also possible. They occur mainly in rather good areas, like in the clipped region in mapping (c1), but also in some formerly rather bad regions as, for example, at the top of picture (c1) or in the middle of the top part of mapping (c2). After the hydrogen treatment lifetime values above $300 \mu \mathrm{s}$ have been detected in string ribbon silicon as well as in the clipped region of the EFG picture (c1). Finally, lifetime measurements after ten hours of illumination under one sun have revealed the MIRHP passivation to be stable under illumination within the range of the measurement technique's accuracy.

A very important aspect visible in Fig. 2 is that regions of comparable asgrown lifetimes do exist within EFG or string ribbon silicon which are influenced differently by the individual processing steps. And such areas have not only been found in those wafers that had undergone P-gettering and hydrogenation but also in those that had been treated according to the other processing sequences given in Fig. 1. The marked region II in the extreme left column (EFG) of Fig. 2, for example, improves significantly during hydrogenation, whereas lifetimes in region III remain rather low, although it has shown the same starting lifetimes and even better lifetime values after P-gettering than area II. Moreover, the marked region $I$ includes an area with about the same starting lifetime as those mentioned before, but which has hardly improved during all the processing steps. Areas of similar behaviour have also been marked in the mappings of the string ribbon wafer (IV, V and VI).

\subsection{Comparison of different processing steps}

A comparison of the various processing steps given in Fig. 1 requires the handling and evaluation of quite a number of lifetime mappings. This has turned out to be a process requiring much involvement. Therefore, the corresponding histograms will be compared in the following section. In this way some of the spatially resolved information gets lost but the histograms still provide much more information than integral measurement values. 
Due to the varying material properties one has to care about the comparability of the different samples before comparing the corresponding histograms. The analysis of the distributions of as-grown lifetimes in the different wafers is a way to find out to which extent the histograms can be compared. The highest degree of comparability, however, is provided by using wafers which once were located next to each other in the direction of ribbon crystallization so that they have nearly the same grain structure. Usually, no ribbons can be found where the grain structure remains the same over a length of more than $5 \mathrm{~cm}$. Moreover, the processing equipment requires $5 \times 5 \mathrm{~cm}^{2}$ sized wafers. Therefore, it is only possible to compare pairs of wafers.

Fig. 3 shows the bulk lifetime distributions in EFG silicon after different processing steps. Each diagram contains the histograms of the $\mu$-PCD measurements of one sequence. The wafers used for investigating sequences 2 and 3 are neighboured ones so that they can be compared to each other directly. Things are the same for the samples of sequences 1 and 4 .

Comparing the histograms after P- and Al-gettering in sequences 2 and 3, one finds a more pronounced gettering effect in the case of Al-gettering. After the subsequent MIRHP passivation, however, these differences are no longer visible. In
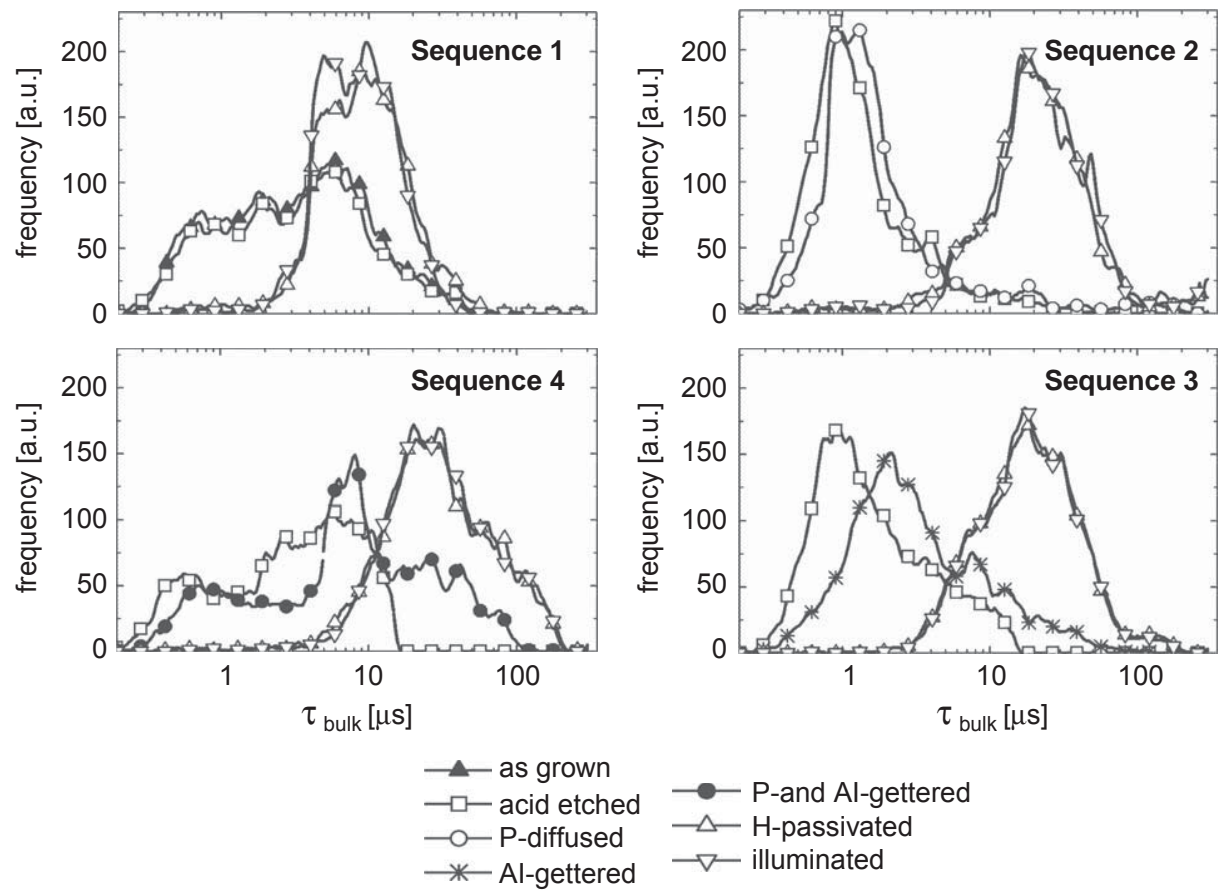

Fig. 3. Histograms of bulk lifetime mappings of EFG silicon after different processing steps. Each of the diagrams contains the lifetime distributions of the $\mu$-PCD measurements that have been performed subsequently on the same wafer according to the schematic drawing in Fig. 1. 
both cases as well as in sequence 4 a pronounced enhancement of bulk lifetimes has been reached by the hydrogenation step. The lifetime values in sequence 1 , instead, are significantly less improved by the hydrogen treatment. Therefore, the MIRHP passivation seems to be more efficient if a gettering step precedes the hydrogenation.

In [6] $20 \mathrm{~min}$ of hydrogen incorporation at $350^{\circ} \mathrm{C}$ have proven to be sufficient for defect passivation in EFG solar cells. In this study the hydrogen passivation lasted six hours at the same temperature, so that a complete defect passivation could be assumed afterwards. Consequently, the differences in passivation efficiency cannot be put down to an accelerated diffusion or a higher hydrogen content in the wafer surface in the case of the gettered wafers. Instead, a synergetic effect between gettering and MIRHP passivation seems to exist, whereas it is of minor importance whether a P- or Al-gettering step or both are performed before the hydrogenation. The underlying effects have not been understood so far. The nature of not hydrogen sensitive defects might be changed during the gettering step in such a way that they can be hydrogenated afterwards, or defects might be generated during gettering which can be passivated again by a subsequent MIRHP treatment.

Bulk lifetimes tend to be unstable under illumination if no gettering step is performed, as can be seen in the corresponding lifetime distributions in the diagram of sequence 1. This might be related to the existence of $\mathrm{Fe}-\mathrm{B}$ pairs which dissociate under illumination so that the resulting interstitial $\mathrm{Fe}$ atoms enhance the recombination [12,13]. In the wafers of sequence 2-4, instead, the possible Fe contaminations have probably been gettered so that this effect is no longer visible.

In the case of string ribbon silicon no adjacent wafers have been used for this investigation. Therefore, the histograms of the lifetime mappings of different processing steps cannot be compared directly. Consequently, the impacts of the various processing steps have been analyzed with the help of the lifetime mappings. The obtained results are the same as those discussed above in the case of EFG silicon.

\subsection{Split mappings}

The non-uniform behaviour of regions with comparable starting lifetimes described previously can be seen more clearly in split mappings. Those of the string ribbon wafer depicted in Fig. 2 are shown in Fig. 4. In this illustration, the lifetime mapping that had been performed before the application of any processing step, i.e. picture (a) which is identical to mapping (a2) in Fig. 2, has been split up into three

Fig. 4. Bulk lifetime mappings of a string ribbon wafer treated according to sequence 2 as obtained after appropriate combination of various measurements. Mappings have been split according to their starting lifetimes. Graphs (a), (ax) and (e) show absolute lifetime values, whereas (bx), (cx) and (dx) illustrate gains or losses caused by each processing step as related to the lifetime values measured before this individual step. 


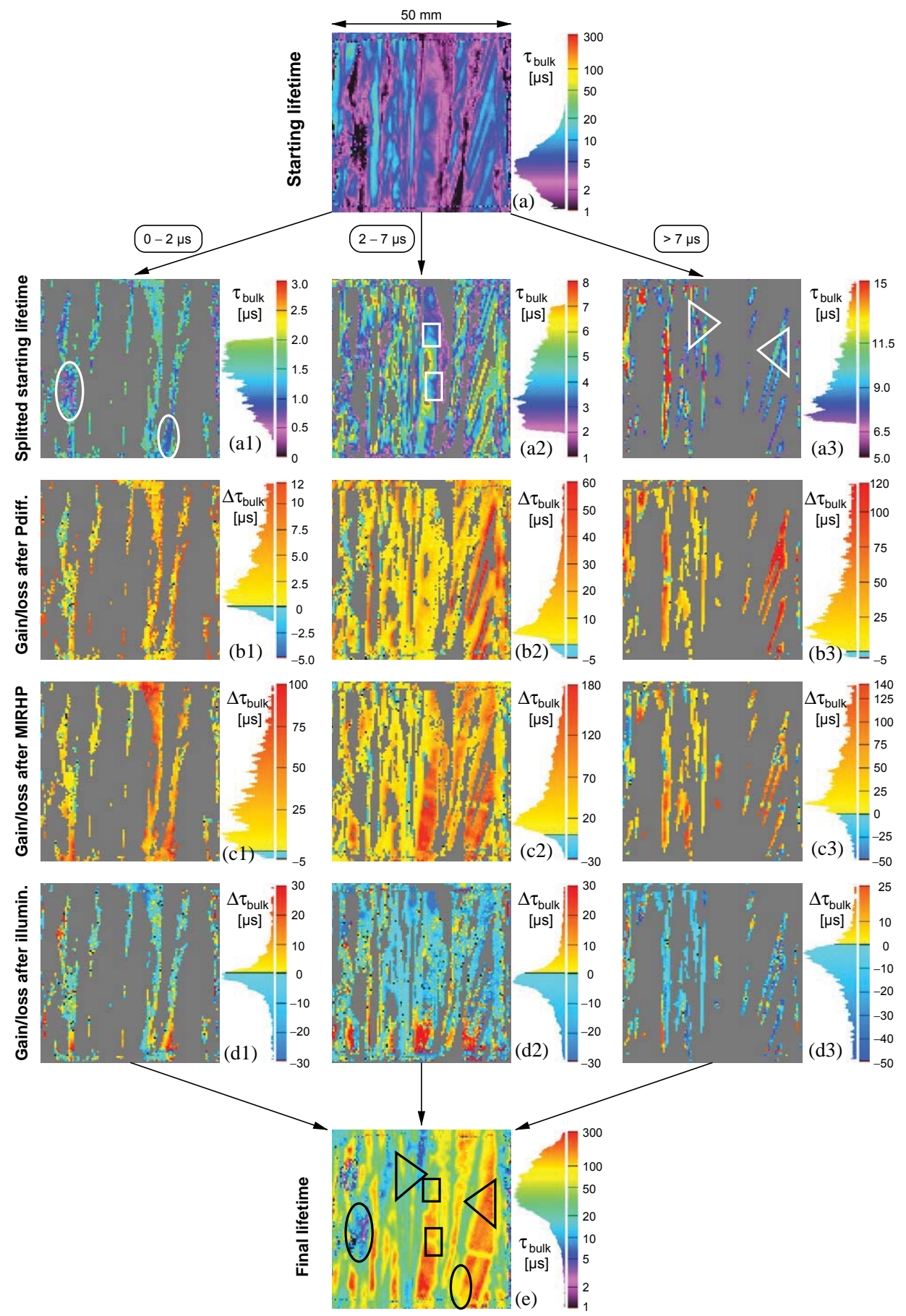


sub-mappings: a first one with lifetime values of up to $2 \mu \mathrm{s}$ (a1), a second one with values between 2 and $7 \mu$ s (a2) and a third one that covers bulk lifetimes of more than $7 \mu$ s (a3). The corresponding columns (a1)...(d1), (a2)...(d2), etc. illustrate the impacts of processing steps on the areas of each lifetime range. The mappings (ax) always show the starting lifetimes before any processing step. $(\mathrm{b} x),(\mathrm{c} x)$ and $(\mathrm{d} x)$, instead, visualize the gains or losses caused by the different processing steps related to the lifetimes measured before the application of such a step. Picture (c1), for example, shows the difference between lifetime values after MIRHP passivation and those after P-gettering for those areas which had a starting lifetime of up to $2 \mu$ s. Finally, mapping (e), which is the same as picture (d2) in Fig. 2, gives the final lifetime values of the whole wafer that had been reached after completion of processing sequence 2 .

When analysing the lifetime alterations after P-gettering by comparing the histograms of mappings (b1) to (b3) in Fig. 4, it seems as if lifetimes are enhanced more efficiently in regions with higher starting lifetimes. The areas with starting values of up to $2 \mu \mathrm{s}$ (b1) are only insufficiently improved by a few microseconds or in the best case by about $10 \mu \mathrm{s}$. In the second column, or the histogram of mapping (b2) respectively, the enhancements have been stronger, but the most striking gains of up to $120 \mu$ s have been reached in the areas with starting lifetimes above $7 \mu \mathrm{s}$ (b3).

That is what things would look like if one just considers the histograms neglecting the spatially resolved information. But things turn out to be more complicated if the corresponding mappings are consulted: there the effect mentioned in Section 3.1, according to which regions of comparable starting lifetimes can react very differently to applied processing steps, is very clearly visible. For example, in picture (b3) in the case of P-gettering lifetime enhancements of hundred or more microseconds are found as well as such which have just improved by 30 to $50 \mu$ s. And similar behaviour occurs in all mappings of row $(b x)$.

Such effects are visible even more strikingly in the split mappings after hydrogen passivation (cx). In each of the mappings, regions can be found where lifetime alterations caused by hydrogenation differ by up to $100 \mu \mathrm{s}$. In order to illustrate this behaviour, areas have been marked in each category which show the same starting lifetime but react differently to the applied processing steps so that the final lifetime values in (e) are strongly varying.

Regions of different behaviour have not only been found in the specific samples of Fig. 4 but also in all wafers of both materials that have been used for the investigation of the different processing sequences given in Fig. 1. Due to their existence and their inhomogeneous distribution according to usual wafer sizes, integral lifetime measurements appear not to be well suited for accurate lifetime analysis. That holds true for EFG silicon as well, where similar results have been obtained. Therefore, spatially resolved measurement techniques should be used for detailed lifetime investigations in EFG or string ribbon silicon instead of integral ones.

With respect to solar cell fabrication, however, the discussed different reactions within regions showing a starting lifetime above $7 \mu$ s are less important because in this case nearly all points exhibit a final lifetime of more than $30 \mu \mathrm{s}$. In case of lower 
starting lifetimes, instead, there are areas which are only insufficiently improved by gettering and hydrogenation, e.g. the one marked by the left ellipse in Fig. 4 which has hardly improved during the different processing steps. Such regions limit the efficiency of the whole solar cell and should consequently be avoided during crystal growth. But to do so one has to find out first, in which way these areas differ from sufficiently improvable ones. The method presented in this study can form the starting point for corresponding investigations as it is now possible to separate regions which can be adequately enhanced during processing from those which cannot. If it should be furthermore possible to reveal the reasons for these differences and, as a consequence, to avoid the growth of wafer areas that improve only weakly, this would result in better solar cell performance.

\subsection{Reliability of mean values}

Split mappings like those given in Fig. 4 have been created not only for the string ribbon wafer that had undergone processing sequence 2 , but also for those of the other sequences illustrated in Fig. 1. The mean lifetimes and standard deviations for every split mapping have been calculated and are shown in Fig. 5a separated according to the different categories of starting lifetime (up to $2 \mu \mathrm{s}, 2-7 \mu \mathrm{s}$, above $7 \mu \mathrm{s}$ ). Additionally, the corresponding mean starting lifetimes of the split mappings are given. In this way it is possible to compare the alterations of mean lifetime that have been caused by different processing steps. The same procedure has been applied to the EFG silicon ribbons and the results for this material are shown in Fig. 5b.

Analysing the data for string ribbon silicon, one finds that the bars look similar to those known from integral measurements [2,4]. But when taking into account the
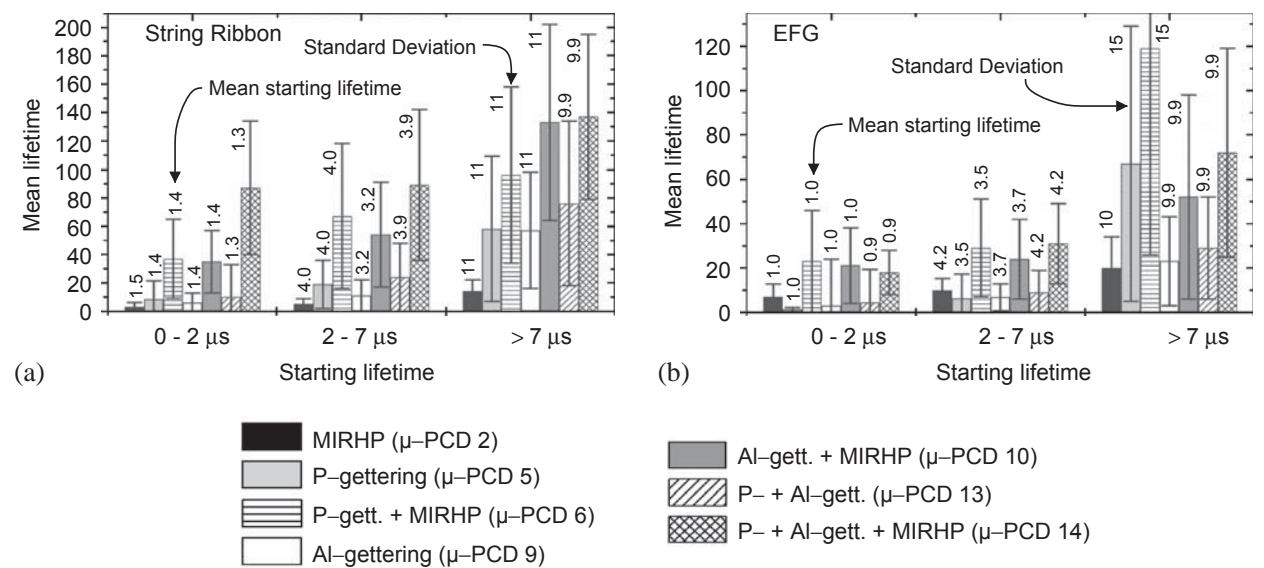

Al-gett. + MIRHP $(\mu-P C D$ 10)

VIIA $\mathrm{P}-+$ Al-gett. $(\mu-\mathrm{PCD} 13)$

P- + Al-gett. + MIRHP ( $\mu-$ PCD 14)

Fig. 5. Mean bulk lifetime values after different processing steps calculated for three regions of different starting lifetimes. The figure at the error bars give the mean starting lifetime in the different split mappings. The $\mu$-PCD numbers in the legend refer to Fig. 1 . 
very large standard deviations, it becomes clear that an enormous amount of information gets lost by the calculation of mean values - despite the fact that the measurements have already been split into three groups of different starting lifetimes. Otherwise the deviations would have been even larger. Consequently, it is difficult to compare the impact of the different processing steps with the help of these mean values alone.

It is worth mentioning again that each standard deviation given in Fig. 5 rises from the variations in lifetime in a certain split mapping of one wafer. This aspect is important as the material quality is also varying from wafer to wafer (see Section 3.1). When performing integral measurements it is therefore reasonable to measure quite a number of wafers in order to take into account these variations as it has been done, e.g. in [14]. But this approach does not take into account the variations within one measured sample (see Fig. 2), as it is not clear in which way the "mean" value delivered by an integral measurement technique comes into existence; whether it is an arithmetic or geometric mean or something different. Accurate information can therefore only be provided by the analysis of mappings or split mappings, respectively.

Things are similar for EFG silicon. Again the bars look similar to those known from integral measurements [2,3], but once more there are large standard deviations. Nevertheless, there is one aspect that can be seen clearly in both graphs of Fig. 5: remote hydrogen plasma passivation is much less efficient if no gettering step precedes the hydrogen processing.

\section{Summary}

A new approach for mapping bulk lifetimes within EFG and string ribbon silicon has been presented which allows to cope with measurement problems caused by strongly varying material properties within these silicon ribbons. As a result, the finally obtained mappings show reliable values in the very high as well as in the very low lifetime range.

Different processing steps have been investigated using this technique and the one of P-gettering followed by a microwave-induced remote hydrogen plasma passivation has been discussed intensively. It could be shown for EFG as well as string ribbon silicon that P-gettering leads to strong improvements of up to $100 \mu \mathrm{s}$ in regions with starting lifetimes higher than $7 \mu \mathrm{s}$. For lower starting lifetimes only enhancements of some microseconds have been measured. In contrast to that, the following hydrogenation also improves most poor regions significantly, but again the largest enhancements appear mainly in areas of rather good starting lifetimes. A comparison of the different processing steps revealed Al-gettering to be somewhat more efficient than P-gettering. After hydrogen passivation, however, this difference is no longer visible. Furthermore, a synergetic effect between Al- or P-gettering and remote hydrogen plasma passivation has been found, as hydrogenation is much less efficient if no P- or Al-gettering or both of them precede the hydrogenation step. Moreover, bulk lifetime values after gettering and remote hydrogen plasma 
passivation have been revealed to be stable under illumination. If no gettering step precedes the hydrogen treatment, lifetime values tend to be unstable. This might be due to not gettered iron contaminations forming $\mathrm{Fe}-\mathrm{B}$ pairs which dissociate under illumination and enhance the recombination in the wafer.

Spatially resolved minority charge carrier lifetime measurements in EFG and string ribbon silicon have shown rather strong variations of bulk lifetime within some square centimetres of wafer area. It could be seen that these inhomogeneities can become even more striking after gettering and hydrogenation. Furthermore, it has been found that there exist regions of comparable starting lifetimes in EFG or string ribbon material, which react very differently to the various solar cell processing steps. Some improve rather strongly reaching lifetimes above $300 \mu$ s whereas others are only insufficiently enhanced. These areas appear to be inhomogeneously distributed according to usual wafer sizes. The method for mapping bulk lifetimes introduced in this study, however, offers the possibility to identify wafer areas which cannot be improved sufficiently. As a consequence, these regions can be studied in order to find out the reasons for their problematic behaviour. In the following it might then be possible to prevent their formation during crystal growth with the help of the gained knowledge, finally leading to better solar cell performance.

The influence of the regions with different behaviour and the varying material properties on integral lifetime measurement techniques as well as resulting difficulties in comparability of the results have been discussed. In conclusion, it has been found that spatially resolved measurement techniques are required for accurate analyses of EFG or string ribbon silicon.

\section{Acknowledgements}

This work was partly supported within the KoSi programme by the German Bundesministerium für Wirtschaft under contract number 0329858J.

\section{References}

[1] T.M. Bruton, G. Luthardt, I.A. Dorrity, J. Alonso, K. Declerq, A. Räuber, A. Vallêra, et al, in: Proceedings of the 14th EC PVSEC, Barcelona, Spain, 1997, p. 11.

[2] A. Rohatgi, V. Yelundur, J. Jeong, A. Ebong, D. Meier, A.M. Gabor, M.D. Rosenblum, in: Proceedings of the 16th EC PVSEC, Glasgow, UK, 2000, p. 1120.

[3] J.-W. Jeong, A. Rohatgi, M.D. Rosenblum, J.P. Kalejs, in: Proceedings of the 28th IEEE PVSC, Anchorage, Alaska, USA, 2000, p. 83.

[4] V. Yelundur, A. Rohatgi, J.-W. Jeong, A.M. Gabor, J.I. Hanoka, R.L. Wallace, in: Proceedings of the 28th IEEE PVSC, Anchorage, Alaska, USA, 2000, p. 91.

[5] P. Geiger, G. Hahn, P. Fath, E. Bucher, in: Proceedings of the 16th EC PVSEC, Glasgow, UK, 2000, p. 1214.

[6] P. Geiger, G. Hahn, P. Fath, E. Bucher, Sol. Energy Mat. Sol. Cells 72 (2002) 155-163.

[7] A.G. Aberle, Crystalline Silicon Solar Cells-Advanced Surface Passivation and Analysis, Centre for Photovoltaic Engineering, University of New South Wales, Sydney, 1999. 
[8] K.L. Luke, L.J. Cheng, J. Appl. Phys. 61 (1987) 2282.

[9] A.B. Sproul, J. Appl. Phys. 76 (5) (1994) 2851.

[10] A.W. Stephens, M.A. Green, Sol. Energy Mat. Sol. Cells 45 (1997) 255-265.

[11] M. Spiegel, P. Fath, K. Peter, B. Buck, G. Willeke, E. Bucher, in: Proceedings of the 13th EC PVSEC, Nice, France, 1995, p. 421.

[12] J. Lagowski, P. Edelmann, A.M. Kontkiewicz, O. Milic, W. Henley, M. Dexter, L. Jastrzebski, A.M. Hoff, J. Appl. Phys. 63 (1993) 3043.

[13] J.H. Reiss, R.R. King, K.W. Mitchell, Appl. Phys. Lett. 68 (1996) 3302.

[14] J.W. Jeong, A. Rohatgi, B.R. Bathey, J.P. Kalejs, in: 12th NREL Workshop on Crystalline Silicon Solar Cell Materials and Processes, Beckenridge, USA, 2002, p. 258. 